\title{
Crystals and inflammation
}

\author{
PHILIP PLATT AND W. CARSON DICK \\ From the Department of Rheumatology, University of Newcastle upon Tyne
}

The inflammatory response is one of the most fundamental defence mechanisms in human biology and is considered under the headings 'acute' and 'chronic'. Acute inflammation is associated with small molecular weight mediators such as the biogenic amines, products of the lipoxygenase and cycloxygenase pathways and small peptides such as kinins. The cells commonly considered under this heading are polymorphonuclear leucocytes, eosinophils, and perhaps basophils and mast cells. It is customary to think of chronic inflammation in terms of larger molecular weight molecules such as acidic and neutral proteolytic enzymes, complement component proteins, proteins of the coagulation and fibrinolytic systems, lymphokines, interleukins, and immunoglobulins and to visualise the cellular involvement in terms of lymphocytes, cells of the mononuclear phagocyte lineage, endothelial cells, and fibroblasts.

That such a convenient but artificial compartmentalisation is incorrect is abundantly clear to any histopathologist accustomed to viewing damaged human tissue where these processes may all be seen in the same histological section. When one appreciates the additional confusion of the dynamism of events in human disease, the rate of flow or traffic of incoming cells through an inflammed site, then a simplistic view of inflammation becomes untenable. It is with this insecure backcloth in mind that we have to view the questions currently being asked about the role of crystals in the promotion of inflammation.

What are the cellular and extracellular events and biochemical processes which occur when a crystal interacts with a biological membrane? To begin to think about this it is necessary to consider our present concept of the cell membrane at which point so many important events occur. The lipid bilayer is a three dimensional network which is continuously recycling. Surface membrane fragments are in equilibrium with membrane segments within the cell so that in many circumstances these may be freely interchangeable. Thus the nuclear membrane is well adapted to interchange freely with intracellular structural membrane and with cell surface membrane. It is important to emphasise the fluidity of the membrane; an insulin or antigen receptor may be present and expressed at one time of day yet not at another. Hydrophilic protein segments float in constrained fashion within the lipid bilayer. The glycosylated end of the molecule is orientated towards the inside of the cell, while the superficial polysaccharide segments project into the extracellular milieu. All of the membrane functions which are taken for granted and too often studied in isolation occur in an integrated fashion dependent on events occurring close to and also at a considerable distance from the reaction site. Thus oxygen uptake and metabolism are linked to disulphide exchange interactions and to monovalent and divalent cation transport, all of these being required as fuel to provide energy and to maintain the integrity of the intact cell. Hormone recognition and in some instances internalisation and the process of antigen recognition and 'restriction' by the major histocompatability complex are also dependent on the integrity of the membrane and on the availability of energy and substrates. Phagocytosis, endocytosis, 'regurgitation during feeding', migration, blast transformation, and secretion whether it be of hormone, structural protein, leukotriene, immunoglobulin, interleukin or lymphokine are all integrally dependent on these functions.

We have recorded some of the many interactions between crystals and biological membranes.

Mechanisms of crystal-induced
damage

CELLS

Polymorphonuclear leucocytes, endothelial lining cells

One of the earliest contenders as putative effector cells in the production of crystal-induced tissue destruction was the polymorphonuclear leucocyte. It was within or around these cells that crystals were first discovered. ${ }^{1}$ The stage was set, therefore, for a unitary hypothesis that would explain inflammation and tissue destruction in diseases such as gout and calcium pyrophosphate. This was proposed by Hollander and McCarty and was focused on the polymorphonuclear leucocyte as the central cell. It also stated, if only implicitly, that the responsible crystals were formed in the extracellular space. The model was an extension of the 'rupture from within' hypothesis proposed in the context of pulmonary disease produced by silicate crystals. ${ }^{2}$ After ingestion of a non-metabolisable crystal it is suggested that the crystal surface binds to and disrupts the membrane of the secondary phagolysosome-producing release of lytic enzymes within the cell and cell death. Thereafter both the unmetabolised crystal and these same lytic enzymes are released into the extracellular space where both are then in a position to produce further tissue destruction.

Early work by Phelps and McCarty ${ }^{3}$ showed pronounced suppression of inflammation in a canine urate crystal model when neutropaenia was induced by the administration of vinblastine. The inflammatory response was restored by transfusion of normal blood. Unfortunately, vinblastine exerts effects on many 
systems in addition to the polymorphonuclear leucocyte. This criticism is also applicable to work which overinterprets results with cobra venom depleted animals. In an attempt to overcome this problem Chang and Gralla ${ }^{4}$ produced similar results using heterologous antipolymorphonuclear serum, again focusing attention on the requirement for polymorphonuclear leucocytes.

A considerable amount of excellent work was focused on the late enzymatic events that could be responsible for matrix destruction and cell death and it was documented that this train of events could be set in motion during phagocytosis of preformed crystals. Discharge of enzymes possessing maximal activity either at acidic or at neutral $\mathrm{pH}$ was shown to occur during phagocytosis and endocytosis even in the intact cell and was described as 'regurgitation during feeding'. It was also shown that these cellular enzymes possessed all of the various activities required to digest each of the components of normal or abnormal connective tissue collagen, glycoprotein and proteoglycan matrix. It seems likely that these enzymes exert their maximum effect in the pericellular area or intracellularly rather than in plasma, synovial fluid, or the extracellular space.

A considerable volume of work published subsequently is in accord with this model. Hoffstein and Weissman ${ }^{5}$ exploited the peculiar characteristics of the large lysosomes of the smooth dogfish shark in a most elegant series of studies. The time course of cell death after exposure to preformed crystals was monotored. Cell death began within 10 to 15 minutes of fusion of lysosomes with crystal-containing phagosomes, primary crystal-free phagosomes remaining unaffected. The early release of the cytoplasmic enzyme lactic dehydrogenase in addition to lysosomal enzymes is an indication of the peculiarly destructive effects of some crystals, particularly monosodium urate, on polymorphs. This response was inhibited by cytochalasin B, which impairs phagocytosis, and by agents which increase cyclic adenosine monophosphate activity such as theophylline. These results were consistent with the Hollander and
McCarty 'suicide-sac' hypothesis. A considerable number of published reports now derive from sequential studies of the interaction of preformed urate, calcium pyrophosphate, and other crystals with polymorphonuclear leucocytes in vitro to establish the sequence of events which follows contact and subsequent ingestion of crystals by these cells. Differences betwen crystals tend to be quantitative rather than qualitative and may depend on differences in size, surface charge, and interaction with various adsorbed proteins. Again most reports support the validity of the suicide-sac model at least in vitro.

Although much of the work on the interaction of polymorphs and crystals has centred on the release of lysosomal enzymes the first reaction, chronologically, of the polymorph to crystals is the production of oxygen radicals. These toxic oxygen-derived products include superoxide, peroxides, and hydroxyl radicals and their release is triggered within 30 seconds of contact between polymorph membrane and crystals. ${ }^{6} \mathrm{~A}$ series of methods of measuring these products have been used including cytochrome reduction, ${ }^{7}$ nitroblue tetrazolium reduction ${ }^{8}$ and luminol-dependent chemiluminescence ${ }^{6}$ and have demonstrated modifying effects of protein coating of crystals and complement activation. ${ }^{7}$ The exact role of these potential toxic products is at present unclear, although if nothing else they do offer a very early marker of polymorph activation.

Much of the core of these ideas may stand the test of time and subsequent experiment but at the moment certain observations militate against the simple assumption that the polymorphonuclear leucocyte is indeed the sole cell responsible. Schumacher et al. ${ }^{9}$ have documented clearly in thorough sequential studies that the first cell to interact with a monosodium urate crystal is the synoviocyte and that only thereafter were polymorphonuclear leucocytes recruited.

Glatt et al. ${ }^{10}$ in parallel studies but using the rat pleural cavity, demonstrated only $50 \%$ reduction in inflammatory response when neutropaenia was induced with cyclophosphamide and concluded that the pleural lining cells made an important contribution. The same criticism that may be levelled against the selectivity and specificity of cobra venom or vinblastine is applicable to cyclophosphamide.

Finally, crystal-induced tissue destruction and inflammation has been recorded in man at a time when there could have been only a very few polymorphonuclear leucocytes present. ${ }^{11}{ }^{12}$ These observations do not contradict most of the previous in vitro work but they do focus attention on the importance of cells other than polymorphonuclear leucocytes in the production of inflammation and tissue destruction in vivo.

\section{Platelets}

Platelets are capable of phagocytosing monosodium urate crystals ${ }^{13}$ and respond in two phases, an active phase with release of adenosine diphosphate and triphosphate and serotonin followed by a lytic phase with release of lactic dehydrogenase. How much this interaction of crystals with platelet membranes contributes to the total inflammatory response has yet to be quantified but again the interaction is modulated by proteins that are adsorbed on to the surface of the crystal (vide infra). An early nonspecific interaction may occur through direct contact and hydrogen bonding. Thereafter more specific interaction occurs between exposed $\mathrm{Fc}$ groups projecting from the crystal surface (to which they are bound by their Fab binding site) and platelet membrane Fc receptors in a manner analogous to the binding of crystals to nucleated cell membranes. If these suggestions are true then there must be considerable differences of binding energy at these sites and this has yet to be demonstrated.

\section{NON-CELLULAR MEDIATORS OF IN FLAMMATION}

\section{Immunoglobulins}

The first group to call attention to the binding of protein to the surface of crystals was Scheelet al. in $1954 .^{14}$ The interaction of crystal surfaces with specific proteins such as complement components and clotting factors is well documented. However, many other 
proteins bind to crystal surfaces. IgG binds in the greatest quantities followed by lysozyme, serum albumin, and ovalbumin in that order. ${ }^{15}$ The functions that are affected by this interaction include electrophoretic mobility, isoelectric point and several titratable groups, and functional changes occur in Hageman factor, complement, and IgG. One of the most interesting points that has been emphasised by Hasselbacher ${ }^{16}$ is that conformational changes occur in the tertiary structure of the adsorbed protein which may have profound effects upon its function.

The most intense interest in crystal-protein interaction is focused on immunoglobulin binding, which seems to occur by the Fab binding site, leaving the $\mathrm{Fc}$ fragment free to interact with any cell or structure which possesses an Fc receptor. It is likely that these subsequent interactions at the Fc site account for a considerable proportion of the biological effects of crystal-protein interaction. Cationic species of IgG are bound preferentially to anionic crystals such as calcium pyrophosphate (CPP), monosodium urate, calcium hydroxyapatite, and amphibole asbestos, whereas the only positively charged crystal, chryositile asbestos, binds anionic IgG. Thus electrostatic bonds are important even if other species of bonding such as Van der Waals, hydrophobic or hydrogen bonds may also participate in different circumstances. ${ }^{16}$ These surface charge interactions may dictate the subclass of immunoglobulin bound and even the orientation of the molecule.

It should be emphasised that adsorption of proteins of various species may operate to protect against as well as to enhance membranolysis ${ }^{17}$ and it is probably too early to speculate from the meagre data available on the resultant effect in vivo.

\section{Complement}

There has been considerable interest in the role of complement in the production of crystal-induced diseases. Decreased complement and the presence of complement split products has been noted in acute gout. ${ }^{18}$ In vitro activation of both classic and alternative pathway, with and without the presence of immunoglobulins, has been documented by several authors. Complement activation was at first thought to be produced only when endotoxin was adsorbed to the crystal surface but this hypothesis does not explain all of the experimental data. Subsequent work showed conflicting results due, at least in part, to differences in crystal numbers and surface characteristics on the one hand and other experimental conditions on the other. Naff and Byers ${ }^{19}$ described the activation of complement by monosodium urate crystals. They noted a small decrease in $\mathrm{C} 1$ activity but pronounced decreases in $\mathrm{C} 2, \mathrm{C} 3$, $\mathrm{C} 4$, and $\mathrm{C} 5$ and suggested a mechanism other than the classic pathway of activation. Considerably less activation of complement occurred in agammaglobulinaemic sera. Hasselbacher reported activation of $\mathrm{C} 3$ by monosodium urate, calcium pyrophosphate, and hydroxyapatite, and concluded on the basis of calcium dependency and abolition of the activation with EDT A and EGTA that the classic pathway was the major pathway of activation. ${ }^{20}$ Ginsberg et al. reported decreased $\mathrm{C} 1, \mathrm{C} 4$, and $\mathrm{C} 3$ haemolytic activity and again concluded that the classic pathway was the significant mechanism. ${ }^{21}$ They also suggested that the activation occurred independently of $\mathrm{IgG}$, by direct binding of the crystal to C1q. Doherty and Dieppe reported activation of $\mathrm{C} 3$ in a manner suggestive of alternate pathway activation, and again suggested that the mechanism was independent of immunoglobulin. ${ }^{22}$

These results may be interpreted as showing that crystals alone appeared capable of activating both the alternate and classic pathways, whereas in the presence of adsorbed immunoglobulin activation of the classical pathway by binding through their exposed Fc fragments predominates.

\section{Hageman factor and kinins}

Hageman factor has been suggested as a possible mediator of crystal-induced inflammation through its role in the generation of kallikrein and kinin. Its presence in synovial fluid and activation by monosodium urate crystals has been documented. ${ }^{23}$ Activation of Hageman factor (80 000 Daltons) releases two peptide fragments $(52000$ and 28000
Daltons respectively) and these have been detected after the addition of monosodium urate crystals to abnormal synovial fluid or to dilute normal plasma. Kininogen and pre-kallikrein are necessary co-factors However, an acute gouty arthritis has been found in animals lacking Hageman factor. ${ }^{24}$ These observations suggest that activation of Hageman factor is not a major mediator in crystal-induced arthritis.

There is abundant evidence of activation of the kinin system in vitro but the data is less complete in vivo. It is possible that the kinin system operates in synergy with other inflammatory mediators such as cyclo-oxygenase products to produce the exquisite pain which is so characteristic of podagra. ${ }^{25}$

\section{Acute phase proteins}

The interaction of acute phase proteins and crystals in the pathogenesis of inflammation needs further study, in particular the role of acute phase proteins in mechanisms of termination of the inflammatory response. Information is becoming available on the structure, function, and biosynthesis of acute phase proteins in particular C-reactive protein ${ }^{26-28}$ and alpha-1-acid glycoprotein and the control mechanisms involved. ${ }^{29} \mathrm{Th}$ is information should now be applied to crystal-induced inflammation.

Liposomes, lysosomes, cell membrane fragments, etc.

Crystals of monosodium urate or silica rupture natural and artificial cell membranes in media that do not constrain hydrogen bonding. Lysis is enhanced by the presence in these membranes of androgens such as testosterone and reduced by the incorporation of oestrogens such as $17 \beta$ oestradiol. $^{30}$ It has been suggested that this interaction may contribute to the explanation of the difference in predeliction of the sexes to the development of gouty arthritis.

In summary, the mechanisms of crystal-induced inflammation are complex and almost certainly multifactorial. It is likely that individual crystal types invoke different mechanisms to variable degrees. Furthermore: 'There is no invariant host response to a given 
crystal' (Hasselbacher 1982). Knowledge of mechanisms of crystal-induced inflammation is applicable not only to crystal-induced diseases in rheumatology but also to industrial lung diseases ${ }^{2}$ and possibly atherosclerosis ( $\mathrm{P} N$ Platt and $\mathrm{A}$ Malcolm, p 000).

We believe that the first priority is to accumulate reliable reproducible data designed to dissect the detail of the multiplicity of mechanisms involved in the interaction of different crystal surfaces with different varieties of cell membranes in vitro. Armed with confident and secure data we may then erect testable hypotheses that may be examined in vivo. All of the important methods are available in the laboratory and it might be better to focus attention for a while on this aspect of the subject rather than to continue to erect more and more elaborate models on the present fragmentary and insecure data.

\section{References}

1 McCarty D J, Hollander J L. Identification of urate crystals in gouty synovial fluid. Ann Intern Med 1961; 54: 452-60.

2 Alison A C, Harington J S, Birbeck M. An examination of the cytotoxic effects of silica on macrophages. J Exp Med 1966; 124: 141-61.

3 Phelps P, McCarty D J. Crystal induced inflammation in canine joints. II Importance of polymorphonuclear leucocytes. J Exp Med 1966; 124: 115-25.

4 Chang Y H, Garalla E J. Suppression of urate crystal induced canine joint inflammation by heterologous anti-polymorphonuclear leucocyte serum. Arthritis Rheum 1968; 11: 145-50.

5 Hoffstein S, Weissman G. Mechanisms of lysosomal enzyme release from leucocytes. IV interaction of monosodium urate with dogfish and human leucocytes. Arthritis Rheum 1975; 18: 153-65.

6 Platt $P$ N, Jeffery S, Wilson L, Griffiths I D. The interaction of crystals and polymorphonuclear monitored by luminol dependent chemiluminescence. Ann Rheum Dis 1981; 40: 615.

7 Abramson S, Hoffstein S T, Weissmann G. Superoxide generation by neutrophils exposed to monosodium urate: effect of protein adsorption and complement activation. Arthritis Rheum 1982; 25: 174-81.

8 Simchowitz L, Atkinson J P, Spilberg I. Stimulation of the respiratory burst in human neutrophils by crystal phagocytosis. Arthritis Rheum 1982; 25: 181-8.

9 Schumacher H R, Phelps P, Agudelo C A. Urate crystal induced inflammation in dog joints: sequence of synovial changes. J Rheumatol 1974; 1: 102-13.

10 Glatt M, Dieppe P A, Willoughby P A Crystal induced inflammation, enzyme release and the effects of drugs in the rat pleural space. J Rheumatol 1979; 6: 251-8.

11 Ortel R W, Newcombe D S. Acute gouty arthritis and response to colchicine in the virtual absence of synovial fluid leucocytes. $N$ Engl J Med 1974; 290: 1363-4.

12 Matthay M, Lindamood M, Steigerwald J C. Acute pseudogout in the absence of synovial fluid leucocytes. $J$ Rheumatol 1977; 4: 303-6.

13 Schumacher H R, Phelps P. Sequential changes in human polymorphonuclear leucocytes after urate crystal phagocytosis. An electron microscopy study. Arthritis Rheum 1971; 14: 513-26.

14 Scheel L D, Smith B, Van Riper J, Fleischer E. Toxicity of silica; II characteristics of protein films adsorbed by quartz. Archives of Industrial Hygiene 1954; 9: 29-36.

15 Kozin F, McCarty D J. Protein binding to monosodium urate monohydrate, calcium pyrophosphate dihydrate and silicon dioxide crystals. I Physical characteristics. J Lab Clin Med 1977; 89: 1314-25.

16 Hasselbacher P. Crystal-prote in interactions in crystal-induced arthritis. In: Weissmann G, ed. Advances in inflammation research. Vol. 4. New York: Raven Press, 1982.

17 Malawista S E, Van Blaricon G, Crettela S B, Swartz M L. The phlogistic potential of urate in solution; studies of the phlogistic process in human leucocytes. Arthritis Rheum 1979; 22: 728-36.

18 Pekin T J, Zvaifler N J. Haemolytic complement in synovial fluid. J Clin Invest 1964; 43: 1372-82.

19 Naff G B, Byers P H. Complement as a mediator of inflammation in acute gouty arthritis. Studies on the reaction between human serum complement and urate crystals. J Lab Clin Med 1973; 81: 747-60.

20 Hasselbacher P. C3 activation by monosodium urate monohydrate and other material. Arthritis Rheum 1979; 22: $571-8$.

21 Ginsberg M H. The mechanisms of complement activation by monosodium urate. Arthritis Rheum 1979; 22: 612.

22 Doherty M, Dieppe P A, Whicher J. Activation of the alternate pathway complement by monosodium urate monohydrate crystals and other inflammatory particles. Ann Rheum Dis 1983; 42: 285-91.

23 Kellermyer R W, Breckenridge R T. The inflammatory process in acute gouty arthritis. I Activation of Hageman Factor by sodium urate crystals. $J$ Lab Clin Med 1965; 67: 455-60.

24 Spilberg L. Urate crystal arthritis in animals lacking Hageman Factor. Arthritis Rheum 1974; 17: 142-8.

25 Kellermyer R W, Naff G B. Chemical mediators of inflammation in urate gouty arthritis. Arthritis Rheum 1975; 18: 765-70.

26 Volanakis J E, Narkates J. Interactions of C-reactive protein with artificial phosphatidylcholine bilayers and complement. J Immunol 1981; 125: 1820-5.

27 Oliveira E B, Gotschlich E C, Liu T-Y. Primary structure of C-reactive protein. J Biol Chem 1979; 254: 489-502.

28 Mortensen R F, Osmand A P, Lind T F, Gewurz $\mathrm{H}$. Interaction of $\mathrm{C}$-reactive protein with lymphocytes and monocytes complement dependent adherence and phagocytosis.J Immunol 1976; 117: 774-81.

29 Kushner J, Feldmann G C. Control of the acute phase response. J Exp Med 1978; 148: 466-521.

30 Weissmann G, Rita G A. Molecular basis of gouty inflammation; interaction of monosodium urate crystals with lysosomes and liposomes. Nature, New Biology 1972; 240: 167-72. 